\title{
Visible Light Promoted Metal Free Sustainable Reduction of Activated Carbon-Carbon Double Bonds Without Any External Reductant
}

\author{
Anindita Bhowmick, ${ }^{a}$ Prakash K. Warghude ${ }^{\mathrm{a}}$ and Ramakrishna G. Bhat*a \\ aDepartment of Chemistry, Indian Institute of Science Education and Research (IISER)-Pune, Dr. Homi \\ Bhabha Road, Pashan, Pune, 411008, Maharashtra, India
}

\begin{abstract}
Visible light promoted chemo-selective metal free hydrogenation of activated double bond has been achieved via photoredox catalysis. Eosin Y has proved to an efficient catalyst for the effective reduction of C-C double bonds of isatins, oxindoles and maleimides under visible light. The method worked efficiently without the aid of any external reductants. Commercially viable DIPEA has been employed as a sacrificial electron donor and it's in-situ generated cationic radical acts as reductant in this transformation. The method proved to be practical as a broad range of substrates containing activated double bond were easily reduced. The method proved to be scalable on a gram scale and the reduced product has been utilized successfully for the further synthetic application. The systematic and detailed mechanistic studies reveal the reductive quenching of the photocatalyst by the activated double bond.
\end{abstract}

The hydrogenation of unsaturated organic compounds is one of the most important and key transformations in organic synthesis till date. ${ }^{1}$ The hydrogenation is indispensable to synthetic community and this process has been widely employed for a range of applications in the chemical, biochemical, food, agrochemical, polymer and pharmaceutical industries. ${ }^{2}$ The selective reduction is a key process in many biosynthetic pathways. During the biosynthesis of fatty acids, the selective reduction of activated carbon-carbon double bonds is achieved by enoate reductases (in living microorganism) that are abundant in mother nature. ${ }^{3}$ However, in organic synthesis traditionally the hydrogenation reactions are carried out using hydrogen gas in presence of catalytic amount of transition metal or stoichiometric amount of metal hydrides. ${ }^{4}$ In this regard, eco-friendly transition metal-free reduction protocols have been discovered that are much safer than the processes that involve transition metals and reactive hydrogen gas. Hantzsch ester, ${ }^{5}$ silanes ${ }^{6}$ have been used as the reactive organic reducing agent for the hydrogenation of carbon-carbon double bonds. Hydrogenation has also been achieved using frustrated Lewis pairs (FLPs). ${ }^{7}$ There are also examples of chemoselective hydrogenation of unsaturated carbonyl compounds using alkyl phosphines and $N$-heterocyclic carbenes. ${ }^{8}$ However, most of the hydrogenation strategies rely either on the heavy transition metals and hydrogen or on stoichiometric amount of expensive, pyrophoric as well as toxic metal based reducing agents. These perennial problems are still a stumbling block to the safer synthetic world.

Over the last few years the visible light photoredox catalysis has been progressing very rapidly as a promising field in organic synthesis. Photoredox catalysis has been emerging as a safer alternative strategy for the some of the transformations that are tedious to be realised by traditional routes. Very importantly, the visible light mediated transformations are usually non-toxic, eco-friendly and easy to handle with lesser waste. ${ }^{9}$ Very recently, very few visible light-mediated cascade cross-coupling hydrogenation reactions have been reported that avoid the use of any sacrificial oxidants. However, these transfer hydrogenation/semihydrogenation strategies solely rely on the metal complexes under visible light conditions to generate hydrogen in situ. ${ }^{10}$ Despite these advances, a metal free hydrogenation under visible light irradiation is highly desirable and required. 
It is also highly challenging and demanding to enable the selective hydrogenation of electron deficient carbon-carbon double bond in a molecule. Among the molecules containing electron deficient carbon-carbon double bonds, the isatin, oxindole and maleimide based unsaturated compounds have a broad range of application in the organic synthesis as precursors of many natural products and bio active molecules. ${ }^{11}$ In this regard, efforts have been made to reduce the electron deficient or activated carboncarbon double bond selectively by few research groups.

\section{Scheme 1: Methods for the hydrogenation of different electron deficient alkenes}

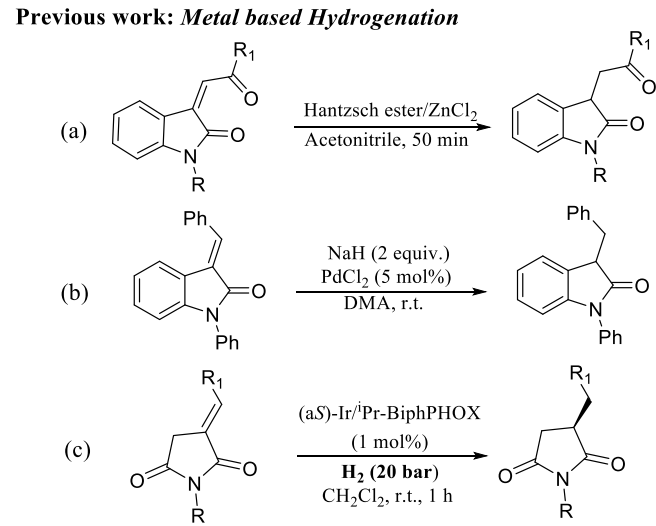

Present Work: Visble light mediated metal-free Hydrogenation

(d)

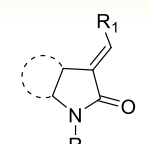
Eosin Y $(10 \mathrm{~mol} \%)$ Blue LED, $60 \mathrm{~W}$ DMSO, $\mathrm{Ar}, 60^{\circ} \mathrm{C}$

$\mathrm{R}=\mathrm{H}$, allyl, propergyl, alkyl, phenyl, benzyl $\mathrm{R}_{1}=$ ketone, aryl, ester, pyridine

Chemoselective reduction Metal free Organophotocatalys External reductant free 28 Examples upto $90 \%$ yield
In 2019, Kasi Viswanath and co-workers described a chemoselective reduction for the isatin and acenaphthenone derived alkene compounds by using Hantzsch ester and $\mathrm{ZnCl}_{2}$ (Scheme 1a). ${ }^{12}$ In the same year, Zhang and co-workers developed a palladium catalysed hydrogenation for the $\alpha, \beta$-unsaturated compounds using sodium hydride as a Michael donor (Scheme 1b). ${ }^{13}$ In 2013, Zhang and co-workers have disclosed an iridium catalysed asymmetric hydrogenation of $\alpha$-alkylidene succinimides derivatives under very high pressure $\left(\mathrm{H}_{2}, 20\right.$ bar Scheme 1c). ${ }^{14}$ However, all of these methods relied on the use of expensive metal catalysts, strong bases or hydrogen gas at very high pressure. The usefulness of these methods are limited due to the drastic reaction conditions and hazard associated with them. It would be really interesting to investigate the reduction of alkenes with electron deficient or activated double bond under completely metal free conditions. It is also important that some of these scaffolds containing activated double bond are routinely explored as intermediates in many transformations and also they are easy to be synthesized for the synthetic applications.
Herein, we wish to report a metal-free sustainable reduction of activated carbon-carbon double bonds in isatin-, maleimide-, 2oxindole derived alkenes under visible light using DIPEA as a reducing agent and Eosin $\mathrm{Y}$ as a photocatalyst (Scheme 1d).

Table 1: Optimization of Reaction Conditions ${ }^{\mathrm{a}}$
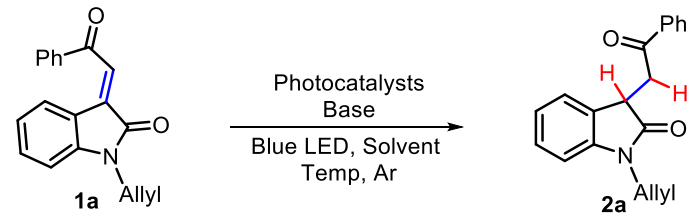

\begin{tabular}{ccccc}
\hline Entry & Photocatalyst & Base & Solvent & Yield (\%) \\
\hline 1 & $\mathrm{Ru}\left(\right.$ bpy) ${ }_{3} \mathrm{Cl}_{2}$ & DIPEA & $\mathrm{MeCN}$ & 50 \\
2 & Eosin Y & DIPEA & $\mathrm{MeCN}$ & 64 \\
3 & 4 CzIPN & DIPEA & $\mathrm{MeCN}$ & 32 \\
4 & Rhodamine 6G & DIPEA & $\mathrm{MeCN}$ & $\mathrm{NR}$ \\
5 & Acridinium & DIPEA & $\mathrm{MeCN}$ & $\mathrm{NR}$ \\
6 & Eosin Y & DBU & $\mathrm{MeCN}$ & $\mathrm{NR}$ \\
7 & Eosin Y & DABCO & $\mathrm{MeCN}$ & $\mathrm{NR}$ \\
8 & Eosin Y & Et ${ }_{3} \mathrm{~N}$ & $\mathrm{MeCN}$ & 46 \\
9 & Eosin Y & DIPEA & DMF & 50 \\
10 & Eosin Y & DIPEA & THF & 18 \\
$\mathbf{1 1}$ & Eosin Y & DIPEA & DMSO & $\mathbf{7 5}$ \\
$12^{\mathrm{b}}$ & Eosin Y & DIPEA & DMSO & 35 \\
$13^{\mathrm{c}}$ & - & DIPEA & DMSO & $\mathrm{NR}$ \\
$14^{\mathrm{d}}$ & Eosin Y & - & DMSO & $\mathrm{NR}$ \\
$15^{\mathrm{e}}$ & Eosin Y & DIPEA & DMSO & NR \\
\hline
\end{tabular}

Reaction Condition: Unless otherwise noted. all the reactions were carried out using $350 \mu \mathrm{mol}$ of 1a, $1.75 \mathrm{mmol}$ of base and 35 $\mu \mathrm{mol}(10 \mathrm{~mol} \%)$ of photocatalyst under blue LED $(60 \mathrm{~W})$ irradiation at $60{ }^{\circ} \mathrm{C}$ for 24 h. ${ }^{b} 10$ equivalent of $\mathrm{H}_{2} \mathrm{O}$ was added to

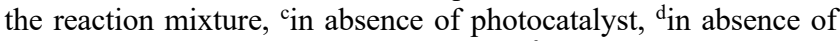
DIPEA, ein absence of light (in dark). 'Isolated yield after the purification by column chromatography. Acridinium $=9$-Mesityl10-methylacridinium perchlorate.

We commenced with the reaction of 1a using DIPEA, in presence of ruthenium catalyst ( $\mathrm{Ru}$ Cat.) in anhydrous acetonitrile under blue LED $(60 \mathrm{~W})$ at $60{ }^{\circ} \mathrm{C}$ using inert atmosphere. ${ }^{15}$ Gratifyingly, we obtained the desired reduction product $2 \mathbf{a}$ in moderate yield (50\%, Table 1, Entry 1). Later, we screened the reaction of 1 a with different photocatalysts in presence of DIPEA (Table 1, Entries 2-5). Among all photocatalysts explored, Eosin Y proved to be most efficient by affording the desired reduced product $\mathbf{2 a}$ in $64 \%$ yield (Table 1 , Entries 2). This result encouraged us to optimize the reaction condition further and in this regard we screened the reaction with different organic bases. We observed that among all the bases, DIPEA proved to be efficient in affording the desired product 2a (Table 1, Entries 2, 6-8). Having optimized the photocatalyst and base, we further screened the reaction using different solvents to enhance the yield of desired product 2a (Table 1, Entries 9-11). Gratifyingly, the reaction worked smoothly in dry DMSO to afford the desired reduction product $\mathbf{2 a}$ in good yield (75\% yield, Table 1 , Entry 11). In order to verify the role of water if any, we carried out 
the reaction of 1a in presence of Eosin Y, DIPEA in DMSO along with $\mathrm{H}_{2} \mathrm{O}$ (10 equiv.). The presence of water reduced the yield of 2a significantly (Table 1, Entry 12). We carried out the optimization further by systematically screening reactions at different temperatures, variable wavelength of visible light (LEDs) and other reaction conditions (see Appendix-I, ESI for details). We further confirmed that photocatalyst, base as well as light are essential for the desired transformation to obtain desired product $\mathbf{2 a}$ (Table 1, Entries 13-15). Based on the exhaustive screening, compound 1a, Eosin Y (10 mol\%), DIPEA (5 equiv.) in dry DMSO under blue light irradiation $(60 \mathrm{~W})$ at $60{ }^{\circ} \mathrm{C}$ proved to be the optimum reaction condition to obtain the desired product $\mathbf{2 a}$ in higher yield.

\section{Scheme 2: Substrate scope of the reduction protocol}

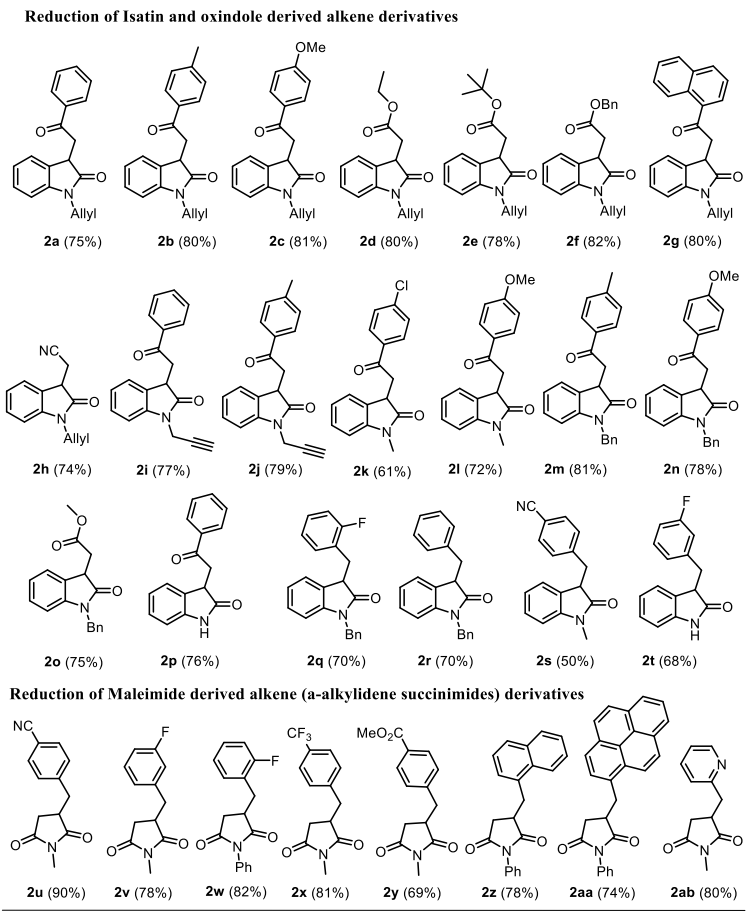

Reaction Condition: Unless otherwise noted. all the reactions were carried out using $350 \mu \mathrm{mol}$ of 1a, $1.75 \mathrm{mmol}$ of base and 35 $\mu \mathrm{mol}(10 \mathrm{~mol} \%)$ of photocatalyst under blue LED $(60 \mathrm{~W})$ irradiation at $60^{\circ} \mathrm{C}$ for $24 \mathrm{~h}$.

Having optimized the reaction condition in hand, we set out to explore the substrate scope of this visible light mediated hydrogenation of electron deficient alkenes. To begin with, we synthesized different isatin based alkene derivatives (1a-1p) to investigate their reactivity and broad substrate scope of the protocol (See ESI S2). We observed that all the substrates (1a-1p) reacted smoothly under the optimized reaction condition to furnish the corresponding reduced products $(\mathbf{2} \mathbf{a}-\mathbf{2} \mathbf{p})$ in moderate to good yields (Scheme 2). It is important to note that all the substrates containing $N$-allyl as well as $N$-propargyl protecting groups reacted easily to afford the corresponding reduced products $(\mathbf{2} \mathbf{a}-\mathbf{2} \mathbf{j})$ in very good yields (up to $82 \%$ yield). Under the reaction conditions, we observed the selective reduction of electron-deficient alkene and the $N$-allyl or $N$-propargyl groups were intact. Moreover, $N$-methyl as well as $N$-benzyl protected isatin derivatives have also reacted efficiently to furnish the desired reduced products (2k-2o) in moderate to good yields (up to $81 \%$ yield, Scheme 2). It is highly remarkable to note that the even the substrate with unprotected nitrogen worked smoothly under the optimized reaction condition to afford the corresponding reduction product in $\mathbf{2 p}$ good yield and we did not observe any side product (Scheme 2).

Later we turned out attention to explore the applicability of the protocol for the reduction of a variety of 2-oxindole based alkenes with different protecting groups as well as electron withdrawing substituents. Under the reaction conditions 2-oxindole based alkenes (1q-1t) furnished the corresponding reduced products (2q2t) in moderate to good yields (up to $70 \%$ yield, Scheme 2 ).

After the initial success, we planned to apply this photocatalyzed hydrogenation protocol for the reduction of $\alpha$-alkylidene succinimides (derived from maleimides). We explored the reactivity of different $\alpha$-alkylidene succinimides (1u-1ab) under optimized reaction condition. We noticed that, all the $\alpha$-alkylidene succinimides (1) $\mathbf{u} \mathbf{- 1} \mathbf{a b})$ reacted smoothly to deliver the corresponding reduction products $(\mathbf{2} \mathbf{u}-\mathbf{2} \mathbf{a b})$ in moderate to very good yields (Scheme 2). It is important to note that, the $\alpha$-alkylidene succinimide derivatives bearing electron withdrawing $\left(\mathrm{CN}, \mathrm{F}, \mathrm{CF}_{3}\right.$, $\mathrm{CO}_{2} \mathrm{Me}$ ) groups, fused aromatic rings and heterocyclic moiety tolerated the optimized reaction conditions to afford the corresponding desired reduction products $(\mathbf{2} \mathbf{u}-\mathbf{2} \mathbf{y}, \mathbf{2 z - 2} \mathbf{a a}$ and $\mathbf{2} \mathbf{a b})$ respectively in moderate to good yields (up to $90 \%$ yield, Scheme 2).

Further, as an extension of this strategy we have performed different transformations using the final products $(\mathbf{2 a}, \mathbf{2 l})$ to demonstrate its synthetic utility (Scheme 3). At first, we have carried out the chemoselective reduction followed by bromination of the ketone moiety of $\mathbf{2 a}$ to obtain the corresponding bromo product 3a (Scheme 3a). Next, we have performed a BaeyerVilliger oxidation of compound $\mathbf{2 l}$ to obtain the corresponding ester $\mathbf{3 b}$ as a final product (Scheme 3b). Further, in order to generalise the practicality of the protocol, gram scale synthesis of $\mathbf{2 a}$ has been achieved ( $0.78 \mathrm{~g}, 67.4 \%$ yield, Scheme $4 \mathrm{a})$.

\section{Scheme 3: Synthetic Utility}
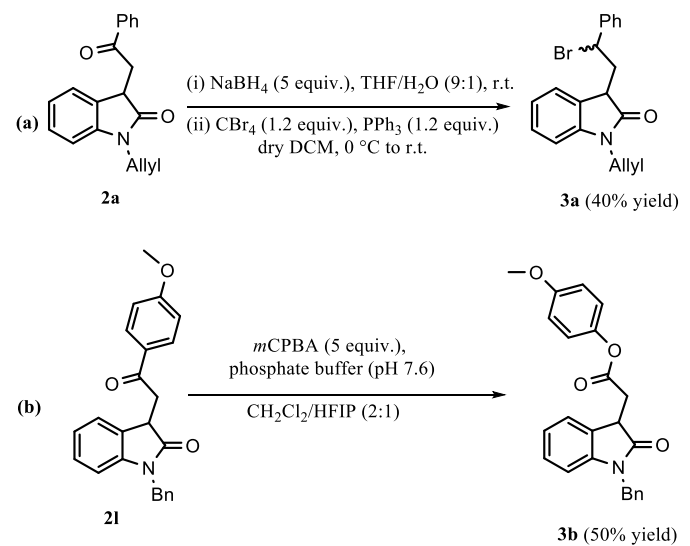
In order to have the mechanistic insight of this transformation, we performed few control experiments systematically. To begin with we carried out a radical quenching experiment of 1a in presence of TEMPO under the optimized reaction conditions. The desired product $\mathbf{2 a}$ did not form even after prolonged reaction time thus supporting the involvement of radical intermediate during the course of the reaction (Scheme 4b). Further to prove the source of hydrogen in the reduction process, we carried out the reaction of 1a under optimized conditions using DMSO- $\mathrm{d}_{6}$ as a solvent (Scheme 4c). However, we did not obtain any deuterated reduced product instead we exclusively obtained 2a and this unambiguously proved that the solvent is not the source of hydrogen (Scheme 4c).

\section{Scheme 4: Controlled experiments and gram scale synthesis}

(a)<smiles>O=C(O)/C=C1/C(=O)N([Al])c2ccccc21</smiles>

(b)

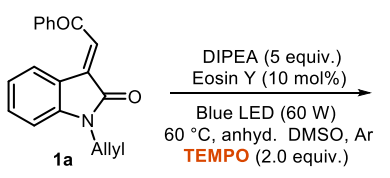

(c)

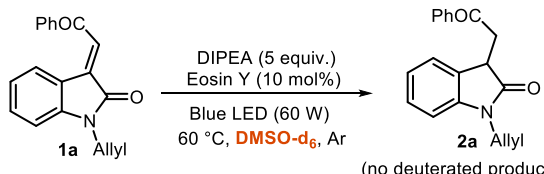

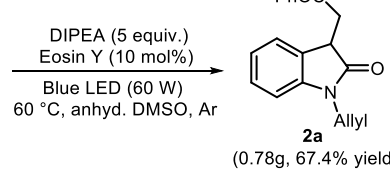

PhOc

(no deuterated product)

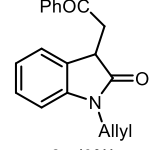

Further, we have carried out fluorescence quenching experiment of Eosin Y in DMSO and 1a (Fig. 1a). Likewise, the fluorescence quenching experiment of Eosin Y in DMSO and DIPEA was carried out under inert atmospheric condition (Fig. 1b).

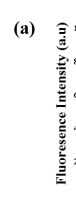

(c)
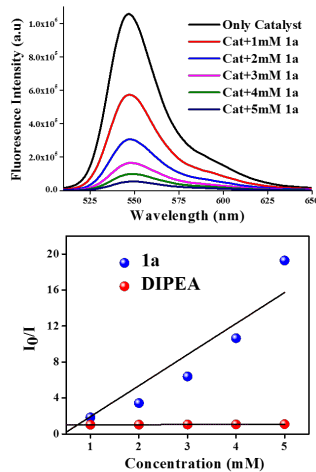

(b)
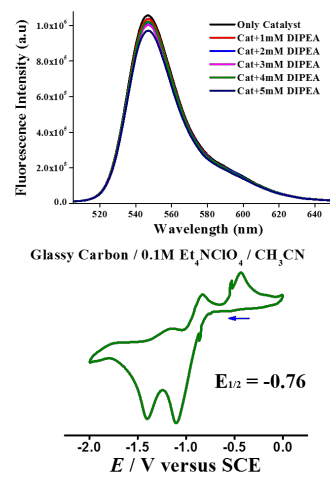

Figure 1: Fluorescence (emission) and cyclic voltammetry studies to have mechanistic insight

(a) Fluorescence quenching experiment of Eosin $\mathrm{Y}$ in presence of 1a (b) Fluorescence quenching experiment of Eosin $\mathrm{Y}$ in presence of DIPEA, (c) Stern -Volmer plot for the fluorescence quenching experiments Eosin Y in presence of 1a and DIPEA, (d) Cyclicvoltammetry plot of $\mathbf{1 a}$.
We observed that the fluorescence emission spectral intensity of Eosin $\mathrm{Y}$ was reduced gradually with the increasing concentration of 1a in DMSO (Fig. 1a). However, we did not observe any significant change in the fluorescence intensity of Eosin $\mathrm{Y}$ in presence of DIPEA (Fig. 1b). These results unambiguously support the oxidative quenching of Eosin $\mathrm{Y}$ in presence of 1a. We have also carried out the cyclic voltammetry of $\mathbf{1 a}$ at room temperature (Fig. 1d). Based on cyclic voltammogram, it was very clear that $E_{1 / 2}$ of $\mathbf{1 a}$ is $-0.76 \mathrm{~V}$ vs. SCE. While P.C (Eosin Y) has a redox potential P.C ${ }^{+} /$P.C $C^{*}=-1.11 \mathrm{~V}$ vs. SCE based on the available data. ${ }^{16}$ It is very clear from the experiments and literature value that 1 a can be easily reduced by the excited state of photocatalyst P.C (Eosin Y).

Based on the control experiment, fluorescence quenching experiments and the cyclic voltammetry study the plausible catalytic cycle has been proposed (Fig. 2). Eosin Y gets excited to Eosin $\mathrm{Y}^{*}$ upon irradiation under visible light. This excited state of the photocatalyst Eosin $\mathrm{Y}^{*}$ gets oxidatively quenched by compound 1a to generate the corresponding $\mathbf{1 a}^{\mathbf{a}^{-}}$radical anion intermediate [as proved by fluorescence quenching experiments (Fig. 1 a,b), SternVolmer plot (Fig. 1c) and cyclic voltammetry]. Further, the oxidized form of the photocatalyst Eosin $\mathrm{Y}^{+}$abstracts an electron from DIPEA (a sacrificial electron donor) to generate the ground state Eosin Y thus completing the photoredox catalytic cycle. Finally, the radical anion intermediate $\mathbf{1 a}^{-}$interacts with DIPEA ${ }^{+}$to $a b-$ stract hydrogen atom by affording the desired product 2a (Scheme 5). ${ }^{17}$

\section{Scheme 5: Plausible mechanistic cycle}

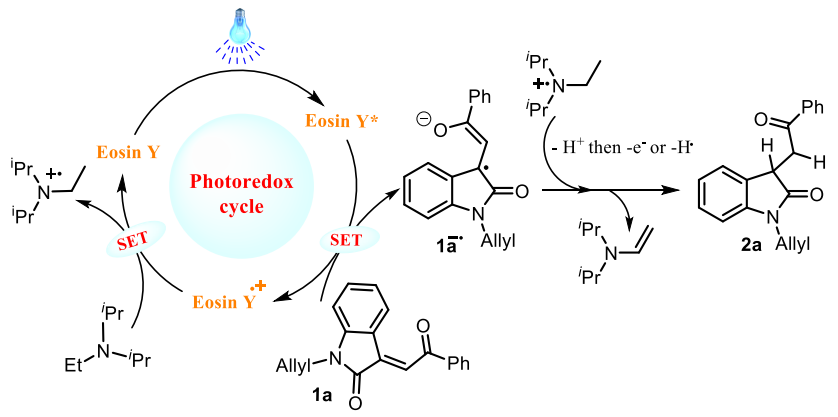

In summary, we have developed a chemo-selective metal free hydrogenation of activated $\mathrm{C}-\mathrm{C}$ double bond of isatin, oxindole and maleimide derivatives under visible light irradiation. The protocol did not require any external reductant for the transformation. Further a detailed mechanistic study revealed the reductive quenching of the photocatalyst by the unactivated double bond. DIPEA has been used as a sacrificial electron donor, and it's in-situ generated cationic radical intermediate acts as a reductant for this transformation. A broad range of substrates were employed in this protocol to obtain the corresponding reduced products in high yields. The method proved to be scalable on a gram quantity and we have also employed reduced products for the further synthetic utility to demonstrate synthetic application. 


\section{ASSOCIATED CONTENT}

\section{Supporting Information}

The Supporting Information with experimental procedures and spectral data are available free of charge-website at DOI:

\section{AUTHOR INFORMATION}

\section{Corresponding Author}

Dr. Ramakrishna G. Bhat- ORCID 0000-0002-7739-9553

*E-mail: rgb@iiserpune.ac.in

Anindita Bhowmick - ORCID: 0000-0002-1367-0578

Prakash K. Warghude - ORCID: 0000-0002-6822-9755

\section{Author Contributions}

\section{ACKNOWLEDGMENT}

R. G. B. thanks Science and Engineering Research Board Department of Science and Technology (SERB-DST), New Delhi, Government of India (File no: CRG/2019/005753), for the generous research grant. A. B. thank CSIR, New Delhi for the fellowship. P. K. W. thanks UGC, New Delhi for the fellowship. Authors also thank Mr. Abhijit Chatterjee, IISER Pune for helping in obtaining the fluorescence spectra and for the useful discussions.

\section{REFERENCES}

1. a) P. Sabatier, M. J. Nye, Chem. World 2004, 1, 46 - 49; b) P. Sabatier, La Catalyse en chimie organique, Paris, Branger, 1913; c) G. Wilkinson, Bull. Soc. Chim. Fr. 1968, 12, 5055 5058.

2. Handbook of Homogeneous Hydrogenation (Eds.: J. G. de Vries, C. J. Elsevier), Wiley-VCH, Weinheim, 2006.

3. a) "Enoate reductase family": S. Steinbacher, M. Stumpf, S. Weinkauf, F. Rohdich, A. Bacher, H. Simon in Flavins and Flavoproteins (Eds.: S. K. Chapman, R. N. Perham, N. S. Scrutton, R. Weber), deGruyter, Berlin, 2002, 941-949. b) R. C. Brewster, J. T. Suitor, A. W. Bennett, S. Wallace, Angew. Chem. Int. Ed. 2019, 58, 12409-12414.

4. a) M. Hudlický, Reductions in Organic Chemistry, 2nd ed., American Chemical Society, Washington, 1996. b) R. Noyori, Angew. Chem. 2002, 114, 2108 - 2123; Angew.Chem. Int. Ed. 2002, 41, 2008 - 2022; c) W. S. Knowles, Angew.Chem. 2002, 114, 2096 - 2107; Angew. Chem. Int. Ed. 2002, 41,1998 2007.

5. a) N. Yutaka, M. Yasuhiko, H. Sawako, O. Akiya, S. Noboru, Bull. Chem. Soc. Jpn. 1989, 62, 1682-1684, b) J. W. Yang, M. T. Hechavarria Fonseca, B. List, Angew. Chem. Int. Ed. 2004, 43, 6660-6662; c) Y. Imada, T. Kitagawa, T. Ohno, H. Iida, T. Naota, Org. Lett. 2010, 12, 32-35, d) M. Sugiura, N. Sato, Y. Sonoda, S. Kotani, M. Nakajima, Chem. Asian J. 2010, 5, 478481, e) X. Xia, Z. Lao, P. H. Toy, Synlett 2019, 30, 1100-1104, f) M. Sugiura, Y. Ashikari, Y. Takahashi, K. Yamaguchi, S. Kotani, M. Nakajima, J. Org. Chem. 2019, 84, 11458-11473.

6. a) M. Rubin, T. Schwier, V. Gevorgyan, J. Org. Chem. 2002, 67, 1936 - 1940; b) J. M. Blackwell, E. R. Sonmor, T. Scoccitti, W. E. Piers, Org. Lett. 2000, 2, 3921 - 3923; c) A. Berkefeld, W. E. Piers, M. Parvez, J. Am. Chem. Soc. 2010, 132, 10660 10661; d) W. E. Piers, T. Chivers, Chem. Soc. Rev. 1997, 26, 345 - 354; e) W. E. Piers, A. J. V. Marwitz, L. G. Mercier, Inorg. Chem. 2011, 50, 12252 - 12262.

7. J. Lam, K. M. Szkop, E. Mosaferi, D. W. Stephan, Chem. Soc. Rev. 2019, 48, 3592-3612.

8. a) M. G. Burnett, T. Oswald, B. J. Walker, J. Chem. Soc. Chem. Commun. 1977, 155-156. b) B. Pal, P. K. Pradhan, P. Jaisankar, V. S. Giri, Synthesis 2003, 1549-1552, c) S.-H. Cao, X.-C. Zhang, Y. Wei, M. Shi, Eur. J. Org. Chem. 2011, 26682672, d) T. Kato, S.-i. Matsuoka, M. Suzuki, Chem. Commun. 2015, 51, 13906-13909.

9. a) K. L. Skubi, T. R. Blum and T. P. Yoon, Chem. Rev., 2016, 116, 10035-10074; b) N. A. Romero and D. A. Nicewicz, Chem. Rev., 2016, 116, 10075-10166, c) M. H. Shaw, J. Twilton and D. W. C. MacMillan, J. Org. Chem., 2016, 81, 6898-6926, d) F. Strieth-Kalthoff, M. J. James, M. Teders, L. Pitzer and F. Glorius, Chem. Soc. Rev., 2018, 47, 7190-7202.

10. a) J.-J. Zhong, C.-J. Wu, Q.-Y. Meng, X.-W. Gao, T. Lei, C.H. Tung and L.-Z. Wu, Adv. Synth. Catal., 2014, 356, 28462852; b) J.-J. Zhong, Q. Liu, C.-J. Wu, Q.-Y. Meng, X.-W. Gao, Z.-J. Li, B. Chen, C.-H. Tung and L.-Z. Wu, Chem. Commun., 2016, 52, 1800-1803.

11. a) F. Zhou, Y.-L. Liu , J. Zhou, Adv. Synth. Catal. 2010 , 352 , $1381-1407$, b) M. Sc. Juliane Schmid M. Sc. Thorsten Junge Dr. Johannes Lang Dr. Wolfgang Frey Prof. Dr. René Peters, Angew. Chem. Int. Ed., 2019, 16, 5447-5451, c) Patrizia Galzerano Giorgio Bencivenni Dr. Fabio Pesciaioli Andrea Mazzanti Dr. Berardino Giannichi Letizia Sambri Dr. Giuseppe Bartoli Prof. Paolo Melchiorre Dr., Chem. Eur. J., 2009, 32, 7846-7849.

12. Gandikota, N.M., Bolla, R.S., Bandyopadhyay, A., Kasi Viswanath, I.V., Russ J Org Chem 2019, 55, 1197-1203.

13. Liu, Y.; Mao, Y.; Hu, Y.; Gui, J.; Wang, L.; Wang, W.; Zhang, S. Adv. Synth. Catal. 2019, 361, 1554-1558.

14. Liu, Y.; Zhang, W. Angew. Chem., Int. Ed. 2013, 52, 22032206.

15. The initial attempts to optimize the reaction conditions at room temperature using different photocatalysts and bases were unsuccessful.

16. Srivastava, V.; Singh, P. P. Eosin Y catalysed photoredox synthesis: a review. RSC Adv. 2017, 7, 31377-31392.

17. (a) Beatty, J.-W.; Stephenson, C.-R. Amine Functionalization via Oxidative Photoredox Catalysis: Methodology Development and Complex Molecule Synthesis. Acc. Chem. Res. 2015, 48, 1474-1484. (b) Schmallegger, M., Gescheidt, G. Benzil/triethylamine: a photo-reducing system for $\mathrm{Cu}^{2+}$. Monatsh. Chem. 2018, 149, 499-504. 